\title{
SPEAR 3 UPGRADE PROJECT: A STATUS REPORT ${ }^{\dagger}$
}

\author{
W. Corbett, R. Hettel, R. Akre, P. Bellomo, R. Boyce, L. Cadapan, R. Cassel, B. Choi, \\ D. Dell'Orco, I. Evans, T. Elioff, R. Fuller, N. Kurita, J Langton, G. Leyh, C.Limborg, D. Macnair, \\ D. Martin, E. Medvedko, C. Ng, Y. Nosochkov, J. Olsen, M. Ortega, C. Pappas, S. Park, \\ H. Rarback, A, Ringwall, P. Rodriguez, J. Safranek, H. Schwarz, B. Scott, J. Sebek, S. Smith, \\ J. Tanabe, A. Terebilo, C. Wermelskirchen, R. Yotam, SSRL/SLAC, Stanford, CA 94309, USA
}

\section{Abstract}

The SPEAR 3 upgrade project at SSRL will replace the original FODO lattice with a 234-m, 18-cell DBA lattice with gradient dipoles. The new hardware draws heavily on PEP-II B-Factory technology: a copper vacuum chamber, IGBT power supply technology, and mode-damped rf cavities to reach beam currents up to $500 \mathrm{~mA}$ at $3 \mathrm{GeV}$. First article magnets, supports, girders, vacuum chambers, pumps and RF components have been fabricated and a prototype girder assembly is nearing completion. I\&C systems, radiation shielding and utility upgrades are in progress. In this paper we report on the status of the main accelerator subsystems.

\section{OVERVIEW}

To better serve the expanding SSRL user community, SPEAR is scheduled for a major upgrade in 2003 [1]. The $18 \mathrm{~nm}-\mathrm{rad}, 500-\mathrm{mA}$ SPEAR 3 will provide one to two orders of magnitude higher performance than SPEAR II.

The 4-year, 58 M\$ SPEAR 3 upgrade project is administered by the DOE, with $\sim 50 \%$ joint funding from NIH. It will completely replace the tunnel floor, rafts, vacuum chamber, magnets, RF, power supplies and cable plant in a 6-month shutdown period. Shielding, utilities and other ancillary systems will be modified before the main shutdown period. A modified transport line and new

Table 1: Parameters for SPEAR 2 and SPEAR 3.

\begin{tabular}{|l|c|c|}
\hline & SPEAR 2 & SPEAR 3 \\
\hline Energy & $3 \mathrm{GeV}$ & $3 \mathrm{GeV}$ \\
Current & $100 \mathrm{~mA}$ & $500 \mathrm{~mA}$ \\
Emittance (w/ID) & $160 \mathrm{~nm}-\mathrm{rad}$ & $18 \mathrm{~nm}-\mathrm{rad}$ \\
RF frequency & $358.5 \mathrm{MHz}$ & $476.3 \mathrm{MHz}$ \\
RF gap voltage & $1.6 \mathrm{MV}$ & $3.2 \mathrm{MV}$ \\
Lifetime @ Imax & $30 \mathrm{~h}$ & $>17 \mathrm{~h}$ \\
Critical energy & $4.8 \mathrm{keV}$ & $7.6 \mathrm{keV}$ \\
Tunes (x,y,s) & $7.18,5.28, .019$ & $14.19,5.23, .007$ \\
e- $\sigma(x, y, s)-$ ID & $2.0, .05,23 \mathrm{~mm}$ & $0.43,0.3,4.9 \mathrm{~mm}$ \\
e- $\sigma(x, y, s)-d i p o l e$ & $.79, .20,23 \mathrm{~mm}$ & $.16, .05,4.9 \mathrm{~mm}$ \\
Injection energy & $2.3 \mathrm{GeV}$ & $3 \mathrm{GeV}$ \\
\hline
\end{tabular}

septum magnet enable $3 \mathrm{GeV}$ injection. All magnets, supplies and chamber components are designed to operate at a top energy of $3.3 \mathrm{GeV}(350 \mathrm{~mA})$. Machine and beam parameters for SPEAR 2 and SPEAR 3 are compared in Table 1. More detailed descriptions can found in $[1,2]$.

\section{LATTICE AND BEAM PROPERTIES}

The double-bend achromat (DBA) lattice will maintain the present beam line alignment for twelve 3.1-m straight sections while providing four 4.8-m straights and two 7.6$\mathrm{m}$ straights. Moderately low $\beta$-functions in the straights $\left(\beta_{\mathrm{x}}=10.2 \mathrm{~m}, \beta_{\mathrm{y}}=4.7 \mathrm{~m}\right)$ provide small beam size $\left(\sigma_{\mathrm{x}}=430\right.$ $\left.\mu \mathrm{m}, \sigma_{\mathrm{y}}=30 \mu \mathrm{m}\right)$ without jeopardizing dynamic aperture or injection. Peak $\beta$-functions below $20 \mathrm{~m}$ are maintained throughout the ring to reduce pumping requirements and sensitivity to field errors. Although the dispersion-free straights eliminate emittance growth from high-field IDs, reductions in $\beta_{\mathrm{x}}$ and $\varepsilon_{\mathrm{x}}$ are possible in the finite-dispersion configuration. Vertical $\beta$-functions in the range $\beta_{\mathrm{y}}=2 \mathrm{~m}$ are possible in the $7.6 \mathrm{~m}$ straights for future IDs [3].

The use of gradient dipole magnets in the arc cells creates a compact lattice, controls peak $\beta_{\mathrm{y}}$ in the dipoles, separates $\beta$-functions at the SD sextupoles and reduces $\beta$ functions in the straights. The remaining magnets were designed with comparable field gradients to facilitate energy ramping.

Betatron phase advances of $\phi_{\mathrm{x}} \sim 0.75, \phi_{\mathrm{y}} \sim 0.2$ provide cancellation of geometric sextupole aberrations across the standard cells. The phase advance and $\beta$-functions in the matching cells were optimized for large on-energy dynamic aperture. Sextupole strengths in the matching cells were optimised for off-energy aperture. The global tunes $(14.19,5.23)$ were chosen to provide robust aperture as a function of tune and to reduce sensitivity to resistive wall impedance. Tracking with magnet errors, ID aberrations and up to $3 \%$ energy oscillations yields a horizontal dynamic aperture in the ID straights of $\sim 18 \mathrm{~mm}$ $\left(A_{x}=32 \mathrm{~mm}-\mathrm{mrad}\right)$. Vertical aperture is limited by $+/-6$ $\mathrm{mm}$ ID chambers ( $1 \%$ coupling, $\mathrm{A}_{\mathrm{y}}=7.6 \mathrm{~mm}-\mathrm{mrad}$ ). Vertical coupling is adjusted using 14 skew quadrupoles.

The projected beam lifetime is $18 \mathrm{~h}$ (28 h gas scattering at $2 \mathrm{nTorr} \mathrm{N}_{2}$-equivalent press, $53 \mathrm{~h}$ Touschek scattering at $500 \mathrm{~mA}$ ). The Touschek value assumes 279 of 372

\footnotetext{
${ }^{\dagger}$ Work supported in part by DOE Contract DE-AC03-76SF00515 and Office of Basic Energy Sciences, Division of Chemical Sciences.
} 
bunches are filled, 3.2 MV RF gap voltage, 3\% energy acceptance and $1 \%$ coupling. The 93 -bucket gap provides ion clearing. On-energy top-off injection can be used to maintain high average beam current.

For beam stability, a $100-\mathrm{Hz}$ closed-loop orbit feedback system is planned. The goal is to reach transverse orbit stability of $20 \mu \mathrm{m}$ horizontally and $5 \mu \mathrm{m}$ vertically on a time scale of several hours at stable BPM sites. Coherent longitudinal bunch oscillations must held to be $<\sim 0.3^{\circ} \mathrm{rms}(\mathrm{dE} / \mathrm{E}<0.01 \%)$ to maintain $<10 \%$ position stability at dipole source points and to provide $<10^{-4}$ energy resolution on undulator harmonics.

\section{MAGNETS AND SUPPORTS}

The SPEAR 3 magnet lattice has $36 \mathrm{C}$-shape gradient dipoles $\left(\mathrm{k}=-0.33 \mathrm{~m}^{-2}\right), 94$ Collins-type quadrupoles, 72 closed-yoke sextupoles, 54 horizontal and 54 vertical combined function dipole correctors $( \pm 1.5 \mathrm{mrad}$ horizontal and $\pm 0.75 \mathrm{mrad}$ vertical). The magnet cores are made with AISI 1010 steel laminations with chamfered ends to improve integrated field quality. The complete magnets are being fabricated by IHEP in Beijing which has so far completed $\sim 20$ dipoles, $\sim 40$ quadrupoles and $\sim 10$ sextupoles (Figure 1), all of which meet or exceed field quality specifications. A 1.13-m Lambertson septum magnet with $8.79^{\circ}$ vertical bend and $5-\mathrm{mm}$ septum aperture is in the final design phase.

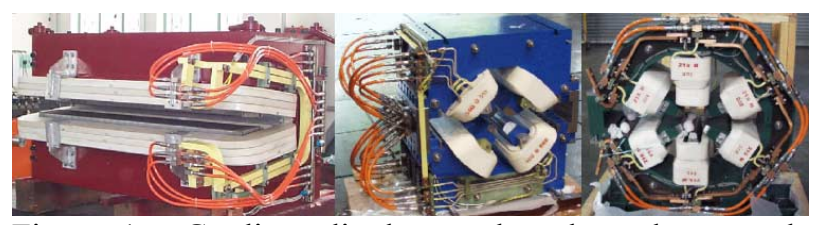

Figure 1: Gradient dipole, quadrupole and sextupole magnets received at SLAC from IHEP, Beijing.

Magnets and vacuum chamber sections will be mounted on 6-strut supports to 3 steel rafts per cell prior to installation. The first prototype raft is nearing completion to check all clearances. Each raft will be rigidly mounted to the new 12" thick concrete floor.

Dipole vibration measurements reveal a longitudinal mode of $17 \mathrm{~Hz}$, attributed to the spring constant of the strut rod ends; all other modes for the dipole and focusing magnets are $>20 \mathrm{~Hz}$.

\section{VACUUM SYSTEM}

To enhance beam stability and protect against highpower radiation strikes, the SPEAR 3 vacuum chamber will be constructed from machined copper plates with discrete photon stops and antechamber geometry. First articles for 2 of the 3 bellows-connected sections have been e-beam-welded at SLAC (Figure 2). Manufacturing and weld distortions in the clamshell halves are minimized by using quarter-hard copper from Revere, Inc. CuproNickel $^{\mathrm{TM}}$ inserts are welded under the corrector

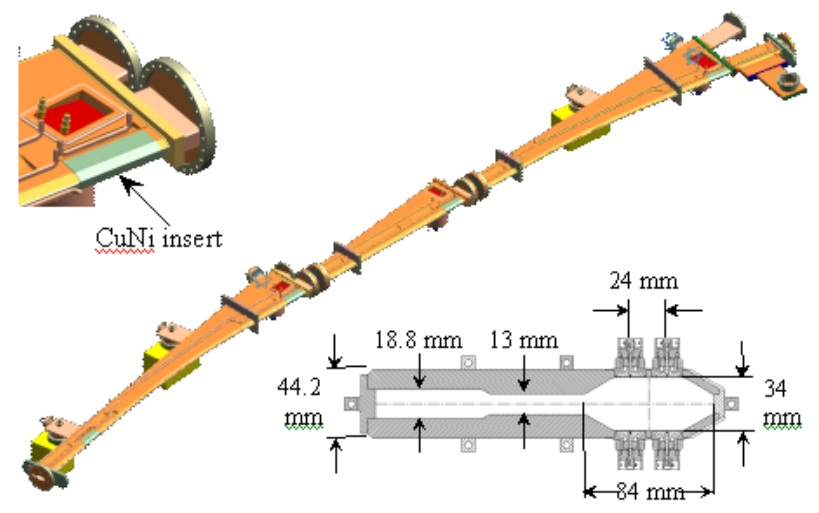

Figure 2: Water-cooled copper vacuum chamber with

cores to increase AC field penetration from corrector magnets to $\sim 100 \mathrm{~Hz}$.

GlidCop ${ }^{\mathrm{TM}}$ masks and crenulated crotch-type absorbers designed to intercept $0.5-8 \mathrm{~kW}$ with power densities up to $20 \mathrm{~W} / \mathrm{mm}^{2}$ and photon fan heights as small as $0.5 \mathrm{~mm}$ are in fabrication at SLAC. Invar supports will restrain vertical vacuum chamber motion at BPM sites to $3 \mu \mathrm{m} /{ }^{\circ} \mathrm{C}$. RF-shielded bellows are in production and $12 \mathrm{~mm}$ BPM buttons have been received from Kaman. The rf impedance of a modified DELTA-style injection kicker [4] is being measured at LBNL. Other chamber components, including DCCT, tune monitor, scrapers and transverse stripline kickers, all preserve the $34 \times 82 \mathrm{~mm}$ chamber cross-section to minimize impedance.

\section{RF SYSTEM}

Four mode-damped PEP II-style RF cavities [5] operating at $476.3 \mathrm{MHz}$ are being fabricated by Accel Instruments, Germany (Figure 3). The new cavities and copper chamber will provide stable operation to $500 \mathrm{~mA}$ at $3 \mathrm{GeV}$. Since the booster frequency remains at 358.5 $\mathrm{MHz}$, it must be phase-locked to enable single-bucket injection. HOM loads, windows, couplers and other components are being built at SLAC.

A PEP-II style, 1.2 MW klystron manufactured by Marconi Applied Technologies, UK, has been delivered to SLAC for testing. At $1080 \mathrm{~kW}$, the tube will drive the 3.2 MV gap voltage required for $500 \mathrm{~mA}$ operation.

The 90-kV, 27-A high voltage supply will be identical to those used for PEP-II. The supply has a SLACdesigned SCR voltage control. The digital EPICS-based

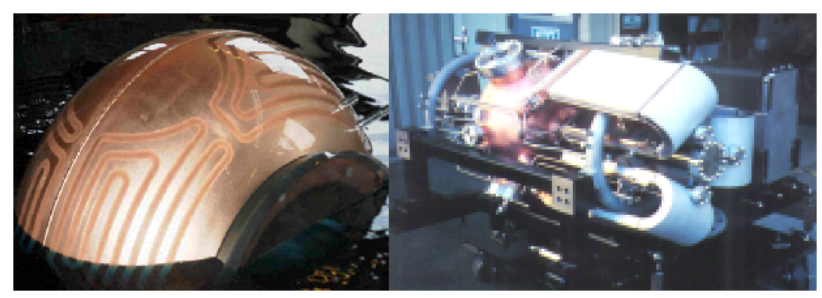

Figure 3: Electroforming copper over RF cavity watercooling passages (left), and a complete cavity assembly. 
PEP-II low-level rf control system [6] is presently being assembled.

\section{POWER SUPPLIES}

The SPEAR 3 power supplies are modelled after the PEP-II design to achieve high performance and integrate maintenance responsibilities at SLAC. IGBT switch-mode unipolar power supplies will be used for all main magnets. The 1-MW dipole power supply $(1.2 \mathrm{kV}, 800 \mathrm{~A})$ will be configured using 4 series/parallel-connected PEP-II chopper modules, each one rated for $600 \mathrm{~V}$ and $400 \mathrm{~A}$. This and other supplies in the $35-135 \mathrm{~kW}$ range employ SCR rectifying bridges to provide soft-start DC voltage to the choppers. 10-ppm stability will be achieved by all main supplies using a SLAC-designed precision regulator unit [7].

IGBT bipolar supplies rated for $\pm 65 \mathrm{~V}$ and $\pm 30 \mathrm{~A}$ are in the construction phase. 108 modules will be used for fast orbit correction ( $\sim 2 \mathrm{kHz}$ supply BW) and 30 for ID trims, skew quads and quadrupole modulation. Each 8-module crate will have a single controller (SLAC design) comprised of a commercial VME CPU serially interfaced over a backplane to digital control daughter boards on the supply modules. 18-bit DACs will minimize correctorinduced noise on the beam. The CPU will provide optional closed-loop control external to internal regulators, and will be interfaced to the control system over a dedicated $100 \mathrm{Mb}$ Ethernet.

IGBT technology is also being used for the 3 induction pulsers used for injection kickers [8]. Each pulser utilizes capacitor-ferrite core sections charged to $\sim 2 \mathrm{kV}$ in parallel and discharged in series to a kicker. The prototype 8-core pulser generates a $16 \mathrm{kV}, 2.5 \mathrm{kA}, 750 \mathrm{~ns}$ wide baseline pulse. Kicker cable length must be $\sim 80 \mathrm{~m}$ to prevent the reflected voltage wavefront from arriving at the pulser before the IGBTs turn off.

\section{INSTRUMENTATION AND CONTROL}

A Channel Access Server has been added to the existing VMS control system to enable use of EPICS tools and applications. Many control and machine modeling applications will use MATLAB ${ }^{\mathrm{TM}}$. Some existing CAMAC systems will be kept, but new processing and interface systems will be configured in VME using PowerPC controllers that serve as EPICS IOCs and DSP engines.

The distributed orbit correction and feedback system, will have a $2-4 \mathrm{kHz}$ cycle rate to achieve a closed-loop bandwidth of $>100 \mathrm{~Hz}$. The feedback system will acquire orbit information from $90 \mathrm{BPM}$ processors in 4 remote processing stations and control 108 correctors. The main orbit acquisition system will employ 54 narrowband switched-button processors. Wideband non-switched processors that can detect first turn and turn-by-turn orbits are also under development. ECDR-814 digital receivers, developed by Echotek according to SSRL specifications [9], will detect the IF from both processor types. A test tone tuned $\sim 640 \mathrm{kHz}$ away from the ring RF will be used to calibrate the parallel-button processors. A quadrupole modulation system will enable beam-based alignment and BPM calibration.

A UV light port is being designed to monitor beam size, bunch length and dynamic beam behavior using fastgated CCD and streak cameras. A GlidCop ${ }^{\mathrm{TM}}$ cold finger will intercept the high-power x-ray beam core and protect a 9-degree flat mirror (rhodium-plated silicon) while permitting most of the UV and visible-light beam to pass. A Schwarzchild reflective focusing optic will be used to form a x2.8 image of the electron beam, which measures $182 \mu \mathrm{m} \mathrm{rms} \mathrm{(H)} \mathrm{x} 51 \mu \mathrm{m} \mathrm{rms} \mathrm{(V)} \mathrm{at} \mathrm{the} \mathrm{dipole} \mathrm{source}$ point, in a diagnostic room outside the ring tunnel. Measurement resolution will be $\sim 20 \mu \mathrm{m}$.

PLC-based interlocks for vacuum chamber protection and magnet systems are being expanded. An orbit interlock using BPMs adjacent to IDs and a secure VME CPU system will be configured to prevent damage to the vacuum chamber from mis-steered photon beams.

\section{BEAMLINE UPGRADES}

Existing bend and wiggler beam lines will be upgraded and optimized for applications exploiting high flux density but requiring only modest beam collimation (many macromolecular crystallography measurements, XAS, etc.). Future ID sources under consideration include a 3.8$\mathrm{m}$ elliptically polarized undulator for soft x-ray (250-2600 eV) applications and 2-m, small gap, in-vacuum undulators for high brightness hard x-ray $(>6 \mathrm{keV})$ applications. These and other sources will exploit the three bend and five insertion device source points that are immediately available in SPEAR 3.

\section{REFERENCES}

[1] "SPEAR 3 Design Report", SLAC Pubs, 1999

[2] R. Hettel et al., "Design of the SPEAR 3 Light Source", Proc. of the 2000 EPAC, Vienna, 625.

[3] Y. Nosochkov, et all, "Study of a Low $\beta_{y}$ Straight Section in SPEAR 3, these proceedings.

[4] G. Blokesch, et al, "A Slotted-Pipe Kicker for High Current Storage Rings", Nucl. Inst. and Meth. In Phys. Res. A 338 (1994) 151-155.

[5] R. Rimmer, et al, "Development of a High Power RF Cavity for the PEP-II B-Factory", Proc of the 1995 PAC, 1792.

[6] P.Corredoura et al., "Low Level System Design for the PEP-II B Factory, Proc. of the 1995 PAC, 2359.

[7] D. Macnair, internal SLAC design report.

[8] R. Cassel et al., "A Solid State Induction Modulator for SLAC NLC", Proc. of the 1999 PAC, 1494.

[9] http://www.echotek.com/. 